\section{Complicaciones asociadas a la broncoscopía flexible: análisis de registro post- procedimiento}

\author{
SEBASTIÁN FERNÁNDEZ-BUSSY ${ }^{1,2}$, GONZALO LABARCA ${ }^{2}$, \\ MÓNICA ZAGOLIN ${ }^{1}$, MAITE OYONARTE ${ }^{1}$, DIONIS ISAMIT ${ }^{1}$, \\ ALFREDO JALILIE $^{1}$, CARLOS GUERRA ${ }^{1}$, SARA CHERNILO ${ }^{1}$
}

\section{Immediate complications following flexible bronchoscopy. Retrospective analysis of 1079 procedures}

\begin{abstract}
Background: Flexible bronchoscopy is a useful diagnostic tool with a relative low rate of complications. Aim: To analyze post procedure risk of complications after flexible bronchoscopy with transbronchial or bronchial biopsy. Material and Methods: The electronic database of a bronchoscopy unit at a general Hospital was analyzed. All procedures performed between 2009 and 2011 were reviewed and complications recorded. The primary outcome measure was the risk for complications defined as the percentage of procedures complicated by hemorrhage, pneumothorax, desaturation $<80 \%$ and other complications. We used a logistic regression model to explore the association between each procedure characteristic and complication. Results: One thousand seventy nine procedures were included in the analysis. Eight percent had complications. Among these, the frequency of hemorrhage was $5.9 \%$ and pneumothorax was $0.3 \%$. Factors associated with complications were exclusive use of topical anesthesia with an odds ratio (OR) of 1.72 (confidence intervals (CI): 1.04-2.86), regular or bad intolerance to the procedure with an OR 4.70 (CI: 3.00-7.38) and performing biopsies of the upper lobes with an OR of 1.76 (CI: 1.04-2.97). Conclusions: Exclusive use of topical anesthesia, performing biopsies of the upper lobes and procedure tolerance were risk factors associated with complications following bronchoscopic biopsies.

(Rev Med Chile 2014; 142: 299-304)
\end{abstract}

Key words: Anestesia; Biopsy; Bronchoscopy. a broncoscopía flexible (FB) es un procedimiento mínimamente invasivo usado ampliamente como herramienta diagnóstica para el estudio de las lesiones pulmonares. Dentro de las múltiples aplicaciones se encuentra la visualización directa de la vía aérea, obtención de muestras para estudio citológico y microbiológico mediante técnicas como el lavado broncoalveolar y cepillado bronquial, y obtención de tejido para estudio histológico a través de distintas técnicas, como la biopsia bronquial (BB) y biopsia transbronquial (BTB) ${ }^{1}$.

Esta herramienta diagnóstica es el procedi-

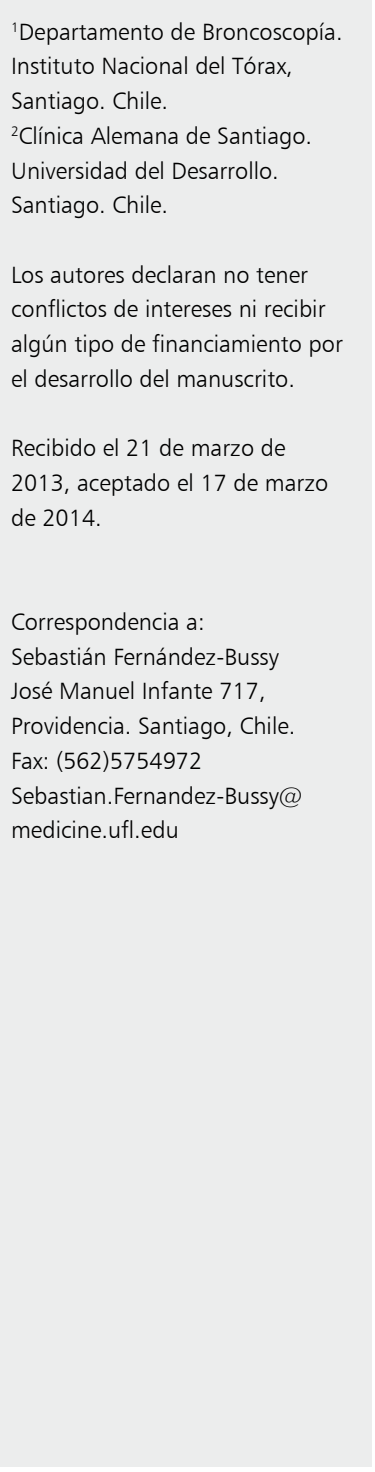

miento de elección para lesiones de vía aérea central $^{1,2}$. Por tratarse de un procedimiento mínimamente invasivo, la FB no está exenta de complicaciones, especialmente relacionadas con la manipulación directa de la vía aérea. El objetivo del presente estudio es describir y analizar el riesgo de complicaciones asociadas a la FB registradas en la base de datos de la Unidad de Broncoscopía del Instituto Nacional del Tórax, centro de referencia a nivel nacional. En especial analizaremos el grupo de pacientes que tuvieron algún tipo de biopsia broncoscópica, ya sea bronquial o transbronquial. 


\section{Materiales y Método}

Se realizó un estudio retrospectivo, mediante a la revisión de la base de datos electrónica de procedimientos realizados en la Unidad de Broncoscopía del Instituto Nacional del Tórax, centro asistencial y docente de referencia a nivel nacional. Se incluyeron los procedimientos realizados entre julio de 2009 y abril de 2011, tanto de pacientes ambulatorios como hospitalizados. Se excluyeron aquellas broncoscopías que no pudieron completarse por mala tolerancia y pacientes con más de un procedimiento durante el período señalado.

Este estudio fue evaluado y aceptado por el comité de ética del Servicio de Salud Metropolitano Oriente de la ciudad de Santiago de Chile.

\section{Protocolo de procedimiento}

Todos los procedimientos fueron realizados por uno de los 6 endoscopistas de planta y un médico en formación con pasantías por broncoscopia por un período de 2 meses continuos. Los procedimientos se realizaron según las recomendaciones estándares, con monitorización y sedación consciente. En nuestro centro los procedimientos son realizados con anestesia tópica con lidocaína al $2 \%$ con o sin sedación mediante la administración endovenosa de midazolam asociada o no a un opiáceo. La vía de ingreso fue la transnasal en la mayoría de los casos, realizándose una inspección completa de la vía aérea y posteriormente en el caso de biopsias transbronquiales, se obtuvo un mínimo de 6 biopsias. No hubo un número mínimo de biopsias bronquiales. La utilización de fluoroscopía fue de acuerdo a la decisión personal del broncoscopista. Al finalizar el procedimiento, se realizó una radiografía de tórax de control en los pacientes que fueron sometidos a BTB sin fluoroscopía y en aquellos pacientes en los cuales se sospecharon complicaciones post-procedimiento. Todos los pacientes firmaron un consentimiento informado en el que autorizaban la realización del procedimiento.

\section{Registro de variables}

Se registraron variables demográficas correspondientes a edad y sexo; se obtuvo información respecto al tipo de procedimiento empleado ( $\mathrm{FB}+\mathrm{BTB}$ o $\mathrm{FB}+\mathrm{BB})$, adicionalmente se obtuvo registro del segmento pulmonar intervenido durante el procedimiento. Respecto al procedimiento se obtuvieron datos sobre la anestesia utilizada, procedimientos guiados mediante uso fluoroscopía, tolerancia del paciente al procedimiento, número de biopsias y complicaciones asociadas al procedimiento. En el caso de la complicaciones severas, se realizó un análisis de la historia clínica con el propósito de obtener antecedentes respecto a la presencia de comorbilidad, lesión pulmonar de base, uso de antiagregantes plaquetarios o anticoagulantes orales e indicación de procedimiento.

Se definieron como complicaciones: hemoptisis leve (sangrado $<50 \mathrm{ml}$ ), hemoptisis severa (sangrado $>50 \mathrm{ml}$ o aquellas que requirieron transfusión de hemoderivados o drogas vasoactivas); neumotórax (de tratamiento médico o con requerimiento de drenaje); desaturación (saturación arterial de oxígeno $<80 \%$ con oxígeno ambiental) y otras complicaciones (vómitos, tos persistente, cefalea, dolor torácico, hipertensión arterial severa).

\section{Análisis estadístico}

Para facilitar el análisis de los resultados, se dividieron las variables en forma dicotómica, de tal manera que las lesiones se clasificaron de acuerdo al procedimiento (BB o TBT), uso de fluoroscopía (sí o no), tolerancia (buena o regular/mala), ubicación (lóbulo superior/medio o inferior), anestesia (tópica o tópica más endovenosa).

Los datos demográficos, clínicos y relacionados con el procedimiento fueron expresados en porcentajes absolutos \pm desviación estándar (DS). Se utilizaron pruebas no paramétricas de U-Mann Whitney para variables continuas y para variables categóricas la prueba de $\chi^{2}$ o prueba de Fischer según corresponda. Se utilizó un análisis multivariado mediante una regresión logística para explorar la asociación entre las distintas variables y el riesgo de tener algún tipo de complicación. Se utilizó un valor $\mathrm{p}<0,05$ para determinar significancia estadística y Odds ratio ajustado (OR) con sus respectivos intervalos de confianza al 95\%. Todos los datos fueron analizados usando el software SPSS 16.0 (SPSS Imc, Chicago, Illinois).

\section{Resultados}

Durante el período en estudio se realizaron un total de $2.317 \mathrm{FB}$ de las cuales 1.350 correspondían a $\mathrm{FB}+\mathrm{BB}$ y $\mathrm{FB}+\mathrm{BTB} ; 271$ registros fueron excluidos por ser procedimientos con registros múltiples. 
La edad promedio (desviación estándar) de los pacientes fue de 64,6 años (DS 13,11 años). Los complicaciones fueron más frecuentes en hombres con 582 casos $(53,9 \%)$ y el promedio de biopsias por procedimiento fue de 6,94 muestras (rango 5-8 muestras).

En relación a los segmentos pulmonares intervenidos, el lóbulo superior derecho (LSD) fue el más frecuente (20,9\%), seguido por el lóbulo inferior izquierdo (LII) con $(18,4 \%)$ y biopsias endobronquiales $(15,4 \%)$. La tolerancia al procedimiento fue buena en $76,9 \%$. El resumen de las características de los procedimientos y las complicaciones registradas se encuentran reflejados en la Tabla 1.

Del total de procedimientos registrados, $7,97 \%$ presentaron algún tipo de complicación. La presencia de hemoptisis leve fue la complicación encontrada con mayor frecuencia con 5,93\% (64/1.079 procedimientos). En $0,27 \%$ (3/1.079 procedimientos) se registró la presencia de neumotórax; mientras que en $0,64 \%$ ( $7 / 1.079$ procedimientos) la saturación arterial de $\mathrm{O}_{2}$ fue menor a $80 \%, 0,27 \%$ (3/1.079 procedimientos) hemoptisis masiva y finalmente $0,83 \%$ (9/1.079 procedimientos) presentaron alguna otra complicación como arritmia benigna, vómitos, hipertensión arterial severa o dolor torácico.

Respecto a las complicaciones graves, en 3 casos se registró la presencia de hemoptisis severa, sin necesidad de transfusiones o uso de drogas vasoactivas, la ubicación fue en LSD en dos ocasiones y en bronquio principal derecho en una ocasión. La anestesia empleada fue tópica en dos casos y endovenosa en uno, la indicación de biopsia fue estudio de neoplasia y no se registró uso reciente de antiagregante o anticoagulante. Del total de casos de neumotórax registrados, dos fueron tratados con instalación de drenaje y evolucionaron sin complicaciones, la ubicación fue en lóbulos superiores en dos ocasiones y en LII en una, la indicación de biopsia fue estudio de neoplasia en dos procedimientos y estudio de enfermedad intersticial en una. No hubo registro de neumotórax a tensión. Todos los casos de neumotórax correspondieron a procedimientos de BTB realizados sin fluoroscopía. Finalmente, no se registraron muertes como complicación del procedimiento.

El análisis univariado de las complicaciones asociadas a los procedimientos mostró que la biopsias obtenidas en pacientes con mala tolerancia al examen tienen un riesgo significativamente mayor comparado con procedimientos con buena tolerancia ( $\mathrm{p}<0,001)$. También demostró que las biopsias tomadas en segmentos pulmonares superiores tienen un riesgo mayor comparado con los segmentos pulmonares medios e inferiores $(\mathrm{p}=0,02)$. No se encontraron diferencias significativas respecto a sexo, tipo de procedimiento (BTB o BB), uso de fluoroscopía y anestesia empleada, variables que podrían estar implicadas en un mayor riesgo de complicaciones.

En el análisis multivariado se observó que las variables que se correlacionan con una mayor probabilidad de presentar algún tipo de complicación fueron el uso exclusivo de anestesia tópica OR 1,72 (IC 1,04-2,86) p 0,04, procedimientos realizados en el los lóbulos superiores OR 1,76 (IC $1,04-2,97)$ p 0,03 , y la tolerancia regular o mala al procedimiento OR 4,70 (IC 3,00-7,38) $\mathrm{p}<0,001$. No se encontraron diferencias estadísticamente

\section{Tabla 1. Fibrobroncoscopía con biopsias bron- quiales y transbronquiales: Características y complicaciones}

\begin{tabular}{|lcc|}
\hline \multicolumn{2}{l}{ n (1.079) } & \% \\
Ubicación & & \\
LSD & 226 & 20,9 \\
LSI & 151 & 14 \\
LM & 146 & 13,5 \\
Língula & 39 & 3,6 \\
LII & 152 & 14,1 \\
LID & 199 & 18,4 \\
Bronquial & 166 & 15,4 \\
Tolerancia & & \\
Buena & 830 & 76,9 \\
Regular & 202 & 18,7 \\
Mala & 47 & 4,4 \\
Complicaciones & & \\
Hemorragia & 64 & 5,93 \\
SaO $<$ 80\% & 7 & 0,64 \\
Neumotórax & 3 & 0,27 \\
Hemoptisis severa & 3 & 0,27 \\
Arritmia benigna & 2 & 0,18 \\
Hipertensión severa & 2 & 0,18 \\
Dolor torácico & 2 & 0,18 \\
Vómitos & 2 & 0,18 \\
Obstrucción bronquial & 1 & 0,09 \\
\hline LD: Lobulo superior derecho; & & \\
\hline
\end{tabular}

LSD: Lóbulo superior derecho; LSI: Lóbulo superior izquierdo; LM: Lóbulo medio; LII: Lóbulo inferior izquierdo; LID: Lóbulo inferior derecho. 
significativas respecto a la edad, género, el tipo de biopsia (bronquial o transbronquial) y el uso de fluoroscopía (Tabla 2).

El análisis de subgrupos según la tolerancia al procedimiento, se encontró diferencia estadís- ticamente significativa en el análisis univariado y multivariado a los procedimientos realizados en mujeres OR 1,77 (IC 1,32-2,38), p < 0,001 y aquellos procedimientos realizados con anestesia tópica OR 1,81 (IC 1,33-2,46), p <0,001 (Tabla 3).

Tabla 2. Análisis univariado y multivariado sobre las variables implicadas en complicaciones secundarias a broncoscopía + biopsia

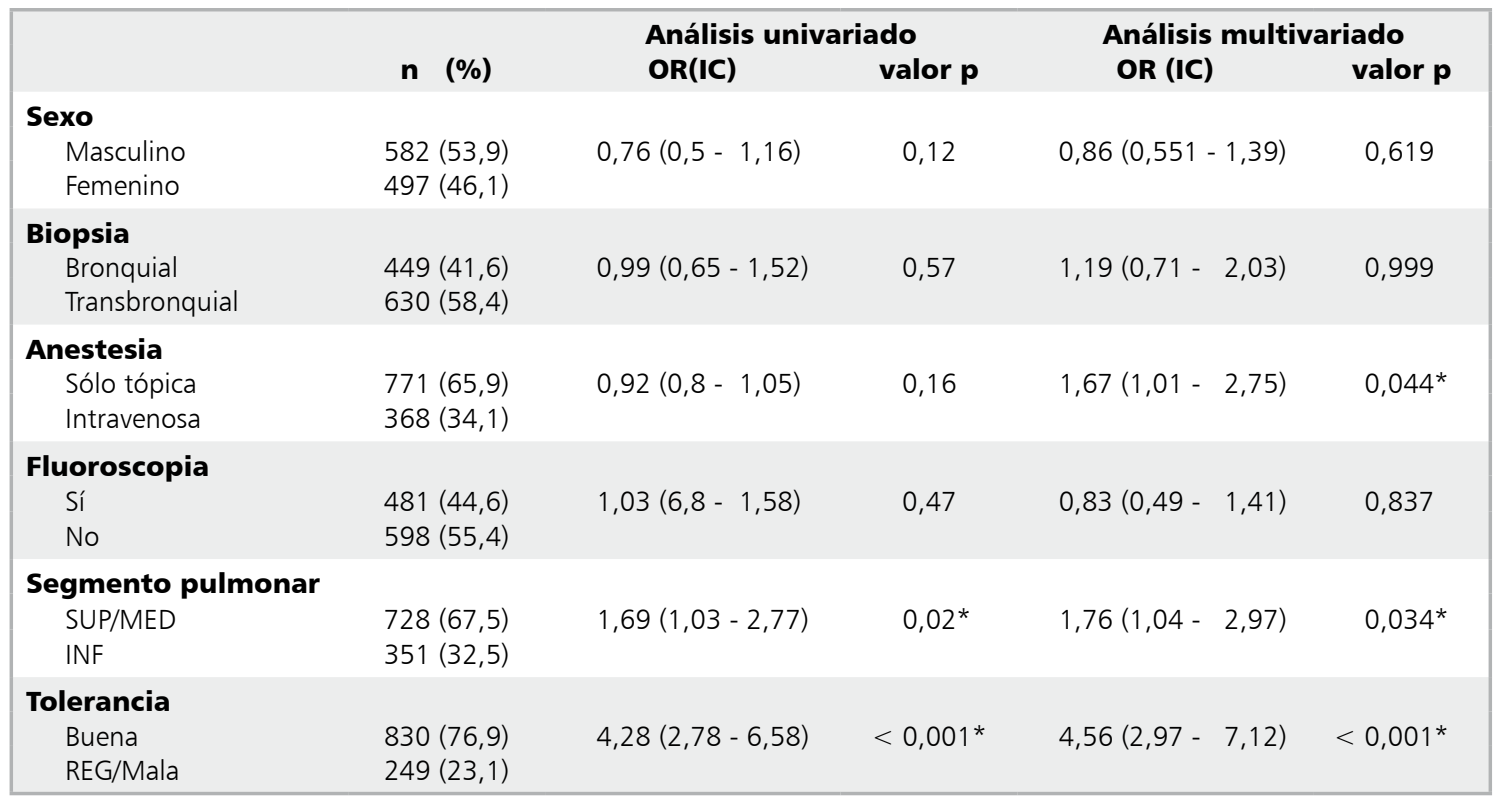

SUP: Superior; MED: Medio; INF: Inferior; Reg: Regular. Se consideró como valor estadísticamente significativo un valor $p<0,05^{*}$.

Tabla 3. Análisis univariado y multivariado sobre las variables implicadas en mala tolerancia al procedimiento

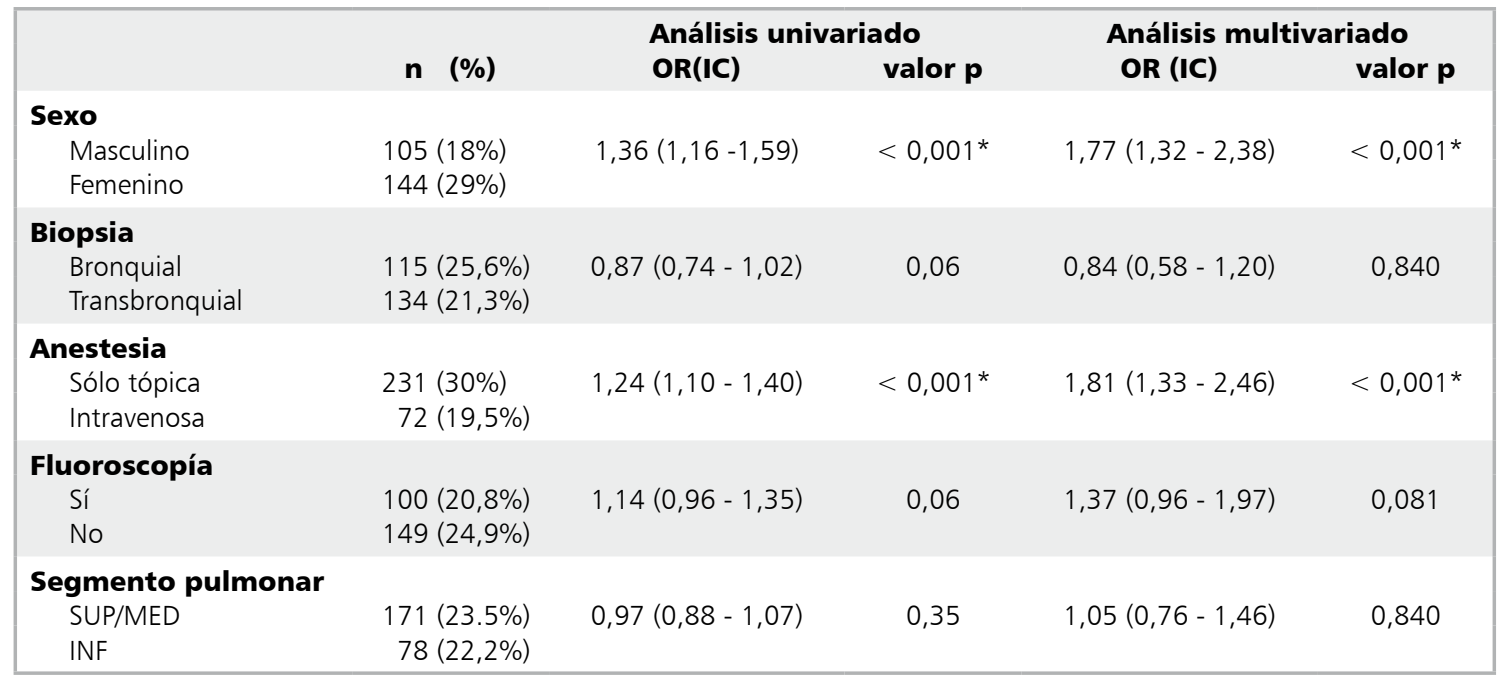

SUP: Superior; MED: Medio; INF: Inferior. Se consideró como valor estadísticamente significativo un valor $p<0,05^{*}$. 
Tabla 4. Complicaciones asociadas a las distintas técnicas broncoscópicas

\begin{tabular}{|lrrc|}
\hline & BTB & BB & valor p \\
Hemoptisis leve & 36 & 28 & 0,878 \\
Hemoptisis severa & 2 & 1 & 0,762 \\
Neumotórax & 3 & 0 & 0,062 \\
$\mathrm{SaO}_{2}<80 \%$ & 5 & 2 & 0,823 \\
Otros & 4 & 5 & 0,998 \\
Total & 50 & 36 & 0,961 \\
\hline
\end{tabular}

BTB: Biopsia transbronquial; BB: Biopsia bronquial.

Finalmente, al analizar las complicaciones asociadas a las distintas técnicas utilizadas (BTB o $\mathrm{BB})$, no se encontraron diferencias significativas, casos de hemoptisis severa fueron secundarias a BTB (Tabla 4).

\section{Discusión}

La BTB y BB son técnicas muy útiles en el estudio de patología pulmonar, sin embargo, como todo método diagnóstico, el riesgo de complicaciones debe ser analizado con el propósito de prevenirlas y evaluar el riesgo versus beneficio asociada a la aplicación de una técnica. La FB es un método diagnóstico que ha demostrado un buen rendimiento con un bajo riesgo de complicaciones $^{3}$. Los distintos reportes de complicaciones asociadas a FB, en especial a aquellos procedimientos en los que se obtiene tejido histológico como la $\mathrm{FB}+\mathrm{BB}$ o $\mathrm{FB}+\mathrm{BTB}$ varían según la metodología empleada (retrospectivo versus prospectivo), período de tiempo evaluado, número de endoscopistas involucrados y número de pacientes evaluados entre otros factores ${ }^{4,5}$.

El porcentaje global de complicaciones reportada en la literatura varía entre $5-7,5 \%$. Las complicaciones menores como la hemorragia leve es la más frecuente con cifras que se encuentran entre $0,12-7,5 \%$. Las complicaciones varían según la técnica empleada, alcanzando las cifras más altas aquellos procedimientos que incluyen obtención de tejido, mientras que técnicas como el cepillado bronquial y el lavado broncoalveolar reportan cifras menores al $1 \%{ }^{4-7}$.

La presencia de complicaciones graves como la hemoptisis severa, neumotórax a tensión o muertes, son las complicaciones más temidas al realizar los procedimientos. Jin y cols en un estudio retrospectivo que incluyó a 23.862 pacientes, analizaron la frecuencia de complicaciones severas en un período de 20 años, encontrando que 152 pacientes presentaron algún tipo de complicación grave, como laringoespasmo o broncoespasmo, los más frecuente, seguido por hemoptisis severa $\mathrm{y}$ arritmias cardiacas ${ }^{8}$.

En relación a las muertes asociadas a los procedimientos, en nuestro centro no se registraron casos durante el período evaluado, cifra concordante con otros reportes, donde los casos de muertes asociadas son inusuales, con cifras que están entre $0,1-0,5 \% 0^{5,7,8}$.

En nuestro centro, el uso exclusivo de anestesia tópica (sin sedación), junto con una mala tolerancia al procedimiento, fueron variables que se relacionan con un mayor riesgo de complicaciones, en especial la hemoptisis, mientras que la sedación intravenosa con midazolam disminuye el riesgo al permitir una sedación más efectiva. Otra variable significativa fueron los procedimientos realizados en los segmentos pulmonares superiores, respecto de las biopsias obtenidas desde lóbulo medio e inferior. Este resultado podría estar sesgado por el mayor número de biopsias bronquiales sobre segmentos superiores, sin embargo, al ser corregido por el tipo de técnica usada, no demostró diferencias entre BTB y BB, lo que demuestra que la instrumentalización de los segmentos pulmonares superiores es una variable que debe ser considerada al momento de realizar el procedimiento. Es probable que la angulación y necesidad de flexionar totalmente el broncoscopio para acceder a los lóbulos superiores, esté relacionado con el mayor número de complicaciones que presentan éstas áreas pulmonares en relación a los campos inferiores, donde se tiene un acceso más directo.

La tolerancia al procedimiento es una variable que influye en la presencia de complicaciones, al analizar las variables implicadas en procedimientos con mala tolerancia, la presencia de anestesia tópica exclusiva, y los procedimientos realizados en mujeres son variables a considerar como potenciales factores de riesgo de complicaciones.

$\mathrm{Al}$ comparar el riesgo de complicaciones asociadas a las distintas técnicas empleadas, no se encontraron diferencias significativas entre la BTB y BB. Tampoco se encontraron diferencias entre 
aquellos procedimientos guiados con fluoroscopía comparado con los no guiados, sin embargo, los pocos casos de neumotórax registrados correspondieron todos a procedimientos trans-bronquiales sin guía fluoroscópica. Este resultado es similar a lo reportado previamente, donde la frecuencia de neumotórax es extremadamente baja, con cifras menores al 1\%. Es importante mencionar que la frecuencia de complicaciones en nuestro centro, no difiere de lo reportado en el extranjero, así como la formación de nuevos broncoscopistas no influye estadísticamente en el porcentaje de complicaciones.

Dentro de las limitaciones de este estudio podemos mencionar que al tratarse de un estudio retrospectivo en el que se analizó la base de datos de la unidad de broncoscopía, no fue posible obtener el total de variables que pueden potencialmente influir sobre las complicaciones, especialmente variables referentes a la ubicación de la biopsia (periferia o central), relación con el mediastino, presencia de comorbilidades, indicación de broncoscopía y variables anatómicas del parénquima pulmonar, como lesiones con bulas, compromiso intersticial o lesiones focales pulmonares. En segundo lugar, este estudio no incluye variables relacionadas con la curva de aprendizaje y entrenamiento de endoscopistas en formación ni variabilidad de cada paciente.

Como conclusión, las biopsias bronquiales y transbronquiales por broncoscopía flexible son un examen relativamente seguro. Dentro de las variables asociadas a una mayor probabilidad de complicaciones, las biopsias de los segmentos superiores, el uso exclusivo de anestesia tópica y la mala tolerancia, variables significativas. Del total de complicaciones la hemoptisis leve es la más frecuente, mientras que el porcentaje de procedimiento que pueden llevar a una complicación grave es baja $(<1 \%)$.

\section{Referencias}

1. Ernst A, Silvestri GA, Johnstone D. American College of Chest Physicians. Interventional pulmonary procedures: Guidelines from the American College of Chest Physicians. Chest 2003; 123 (5): 1693-717.

2. Triller N, Dimitrijevic J, Rozman A. A comparative study on endobronchial ultrasound-guided and fluoroscopicguided transbronchial lung biopsy of peripheral pulmonary lesions. Respir Med 2011; 105 Suppl 1: S74-7.

3. Anders GT, Johnson JE, Bush BA, Matthews JI. Transbronchial biopsy without fluoroscopy. A seven-year perspective. Chest 1988; 94 (3): 557-60.

4. Lukomsky GI, Ovchinnikov AA, Bilal A. Complications of bronchoscopy: comparison of rigid under general anesthesia and flexible fiberoptic bronchoscopy under topical anesthesia. Chest 1981; 79 (3): 316-21.

5. Geraci G, Pisello F, Sciumé C, Li Volsi F, Romeo M, Modica G. Complication of flexible fiberoptic bronchoscopy. Literature review. Ann Ital Chir 2007;78 (3): 183-92.

6. Yung RC. Tissue diagnosis of suspected lung cancer: selecting between bronchoscopy, transthoracic needle aspiration, and resectional biopsy. Respir Care Clin N Am 2003; 9 (1): 51-76.

7. Alzeer AH, Al-Otair HA, Al-Hajjaj MS. Yield and complications of flexible fiberoptic bronchoscopy in a teaching hospital. Saudi Med J 2008; 29 (1): 55-9.

8. Jin F, Mu D, Chu D, Fu E, Xie Y, Liu T. Severe complications of bronchoscopy. Respiration 2008; 76 (4): 429-33. 